\title{
A phase I trial of the selective oral cyclin-dependent kinase inhibitor seliciclib (CYC202; R-Roscovitine), administered twice daily for 7 days every 21 days
}

\section{Benson ',2, J White ${ }^{3}$, J De Bono',2, A O’Donnell', F Raynaud', C Cruickshank ${ }^{4}$, H McGrath', M Walton', P Workman', S Kaye ${ }^{2}$, J Cassidy ${ }^{3}$, A Gianella-Borradori ${ }^{4}$, I Judson $^{*, 1}$ and C Twelves ${ }^{6,7}$}

'Cancer Research UK Centre for Cancer Therapeutics, The Institute of Cancer Research, Sutton, Surrey, UK; ${ }^{2}$ Section of Medicine, The Institute of Cancer Research and Royal Marsden Hospital, Sutton, Surrey, UK; ${ }^{3}$ Department of Medical Oncology and Beatson Oncology Centre, University of Glasgow, Glasgow, UK; ${ }^{4}$ Cancer Research UK Drug Development Office, London, UK; ${ }^{5}$ Cyclacel Ltd, Dundee, UK; ${ }^{6}$ Beatson Oncology Centre, Glasgow, UK

Seliciclib (CYC202; R-roscovitine) is the first selective, orally bioavailable inhibitor of cyclin-dependent kinases I, 2, 7 and 9 to enter clinical trial. Preclinical studies showed antitumour activity in a broad range of human tumour xenografts. A phase I trial was performed with a 7-day b.i.d. p.o. schedule. Twenty-one patients (median age 62 years, range: 39-73 years) were treated with doses of 100, 200 and 800 b.i.d. Dose-limiting toxicities were seen at 800 mg b.i.d.; grade 3 fatigue, grade 3 skin rash, grade 3 hyponatraemia and grade 4 hypokalaemia. Other toxicities included reversible raised creatinine (grade 2), reversible grade 3 abnormal liver function and grade 2 emesis. An $800 \mathrm{mg}$ portion was investigated further in 12 patients, three of whom had MAG3 renograms. One patient with a rapid increase in creatinine on day 3 had a reversible fall in renal perfusion, with full recovery by day 14 , and no changes suggestive of renal tubular damage. Further dose escalation was precluded by hypokalaemia. Seliciclib reached peak plasma concentrations between $\mathrm{I}$ and $4 \mathrm{~h}$ and elimination half-life was $2-5 \mathrm{~h}$. Inhibition of retinoblastoma protein phosphorylation was not demonstrated in peripheral blood mononuclear cells. No objective tumour responses were noted, but disease stabilisation was recorded in eight patients; this lasted for a total of six courses ( 8 weeks) in a patient with ovarian cancer.

British Journal of Cancer (2007) 96, 29-37. doi: I0.1038/sj.bjc.6603509 www.bjcancer.com

Published online 19 December 2006

(C) 2007 Cancer Research UK

Keywords: cyclin-dependent kinase inhibitor; phase I clinical trial

The cyclin-dependent kinases (Cdks) have been reported to play critically important roles in several key cellular processes that are frequently dysregulated in cancer, including cell cycling, transcription and apoptosis (Malumbres and Barbacid, 2001; Benson et al, 2005). The design of small molecule inhibitors of Cdks has, therefore, been actively pursued, and a considerable number of small molecule inhibitors of these serine-threonine kinases have been identified. The first compounds in this class to be evaluated in clinical trials were flavopiridol and the 7-hydroxy derivative of staurosporine, UCN-01 (Senderowicz et al, 1998; Sausville et al, 2001). These first-generation Cdk inhibitors lacked specificity, being broad-spectrum inhibitors that inhibited not only the Cdks but also many other kinases. Second-generation inhibitors were designed to be more selective, with many of these compounds specifically developed to target selected Cdks (Meijer and Raymond, 2003). Seliciclib (CYC202; $R$-roscovitine) is a 2,6,9tri-substituted purine analogue of olomoucine derived from studies evaluating the structure-activity relationships within this

\footnotetext{
*Correspondence: Professor I Judson, Cancer Research UK Centre for Cancer Therapeutics, The Institute of Cancer Research, Sutton, Surrey, UK; E-mail: ian.judson@icr.ac.uk

${ }^{7}$ Current address: University of Leeds, Leeds, UK

Revised 6 November 2006; accepted 7 November 2006; published online 19 December 2006
}

compound class (Meijer et al, 1997; Gray et al, 1998; McClue et al, 2002; Whittaker et al, 2004). The co-crystal structure of seliciclib and Cdk2 has been described, with the drug occupying the ATP-binding pocket of the kinase (De Azevedo et al, 1997).

Seliciclib is a highly selective, orally bioavailable, small molecule inhibitor of several Cdks, competing at their ATP-binding sites (Meijer et al, 1997). It is a potent inhibitor of the human Cdk2/ cyclin E, Cdk1/cyclin B, Cdk7/cyclin $\mathrm{H}$ and Cdk9/cyclin T1 with $\mathrm{IC}_{50}$ s of $0.1,2.7,0.5$ and $0.8 \mu \mathrm{M}$, respectively (McClue et al, 2002). It is, however, only a weak inhibitor of Cdk4, Cdk6 and Cdk8. In a 148 kinase screen, seliciclib was highly Cdk specific, with few other enzymes being inhibited only at micromolar concentrations (Bach et $a l, 2005)$. The latter includes CaM kinase 2, CK $1 \alpha, \mathrm{CK} 1 \delta$, DYRK1A, EPHB2, ERK1, ERK2, FAK and IRAK4. The other kinases tested were insensitive to seliciclib. This screen was not, however, exhaustive, testing only approximately $30 \%$ of known kinases. Affinity purification of proteins bound to $R$-roscovitineagarose beads also identified pyridoxal kinase (PDXK) as a target of seliciclib, binding not at the ATP-binding site but at the pyridoxal site (Tang et al, 2005). Pyridoxal kinase (PDXK) catalyses the phosphorylation of pyridoxine, pyridoxal and pyridoxamine to, respectively pyridoxine- 5 - phosphate, pyridoxal$5^{\prime}$-phosphate and pyridoxamine- $5^{\prime}$-phosphate respectively.

Seliciclib has antitumour activity against a broad range of cancer cell lines (McClue et al, 2002; Whittaker et al, 2004; Raynaud et al, 
2005). Molecular pharmacology studies in human colorectal cancer cell lines have revealed that seliciclib decreases retinoblastoma protein phosphorylation, initially at the Cdk2 phosphorylation site and then at multiple sites (Whittaker et al, 2004). Seliciclib also causes increases in Erk-1 and Erk-2 phosphorylation, although this may have little impact on cell cycling (Whittaker et al, 2004). More importantly, through its inhibitory effects on Cdk7 and Cdk9, seliciclib inhibits RNA polymerase II phosphorylation and total RNA polymerase II protein activity (Whittaker et al, 2004; Raynaud et al, 2005). This results in the decreased expression of several cyclins, including cyclins D1, A and B1, which may explain the observed global effects on retinoblastoma (RB) phosphorylation and the overall cell cycle effects of seliciclib in these models. These include a decreased proportion of cells in G1, decreased DNA synthesis in the S-phase and a moderate increase in cells in $\mathrm{G}_{2} / \mathrm{M}$ (Whittaker et al, 2004; Raynaud et al, 2005).

Other studies report that this agent also blocks the degradation of p53 through the inhibition of MDM2 expression (Lu et al, 2001). Studies in the Lovo colorectal carcinoma cell line indicate that the major effect on these cells is the induction of cell death from all compartments of the cell cycle (Schutte et al, 1997; McClue et al, 2002). The antitumour efficacy of seliciclib has been demonstrated in human tumour xenografts in nude mice, when administered at $500 \mathrm{mg} \mathrm{kg}^{-1}$ twice a day or $200 \mathrm{mg} \mathrm{kg}^{-1}$ thrice a day (McClue et al, 2002; Raynaud et al, 2005). Pharmacokinetic-pharmacodynamic relationships were established in the HCT116 human colon cancer xenograft model and showed reduced phosphorylation of RB and decreased expression of cyclin D1 in tumour tissue (Raynaud et al, 2005). Seliciclib was most active in inhibiting the proliferation of colon, non-small-cell lung, breast and prostate human cancer xenografts. It also induces caspasedependent apoptosis in primary B-cell chronic lymphatic leukaemia cells ex vivo, downregulating the antiapoptotic proteins $\mathrm{Mcl}-1$ and XIAP, and increasing Bak expression and Bax cleavage (Alvi et al, 2005). In addition, seliciclib demonstrates antitumour activity in multiple myeloma cultures and cell lines, inducing apoptosis associated with downregulation of Mcl-1 and interleukin (IL)-6 transcription and protein expression (MacCallum et al, 2005; Raje et al, 2005).

Seliciclib has a satisfactory preclinical pharmacokinetic (PK) profile and is extensively cleared into inactive metabolites, with the carboxylate being the main urinary metabolite after administration to mice and following incubation with hepatic microsomes (Nutley et al, 2005). Seliciclib demonstrated a higher area-under-the-curve (AUC), longer elimination half-life and better intratumoral drug delivery, when compared with the similar 2,6,9-tri-substituted purine Cdk inhibitors olomoucine and bohemine (Raynaud et al, 2004). Seliciclib is highly bound to plasma proteins (90\%), primarily albumin (Vita et al, 2005). To characterise further the PK of this agent, a first in-human, single oral dose, volunteer study was undertaken in 12 healthy subjects, administering between 50 and $800 \mathrm{mg}$ of seliciclib (De la Motte and Gianella-Borradori, 2004). This showed that the parent compound is satisfactorily absorbed, undergoes first-pass metabolism, exhibits high protein binding and is rapidly and extensively distributed into tissues. The carboxylate was confirmed as the main metabolite with low protein binding, limited tissue distribution and renal clearance.

In view of these promising data, seliciclib has been studied in early clinical trials in patients with advanced cancer, utilising several schedules to further evaluate its safety, tolerability and PK properties. The principal objectives of this phase I trial of seliciclib administered orally twice a day for 7 days every 21 days were (1) to determine the toxicity profile of seliciclib (2) to identify the dose limiting toxicities of seliciclib, (3) to establish the recommended dose of seliciclib on this schedule, (4) to characterise the PK behaviour of seliciclib and (5) to document possible anticancer activity in patients with advanced solid malignant diseases.

\section{PATIENTS AND METHODS}

\section{Patient selection}

Patients with a histologically confirmed diagnosis of a malignant solid tumour, refractory to conventional treatment were eligible for treatment, provided they met the following criteria: age $\geqslant 18$ years; World Health Organization (WHO) performance status $\leqslant 2$; estimated life expectancy $\geqslant 3$ months; no previous anticancer therapy within 4 weeks; no significant gastrointestinal disease influencing drug absorption; adequate hematopoietic, hepatic and renal functions $\left(\mathrm{Hb} \geqslant 9 \mathrm{~g} \mathrm{dl}^{-1}, \mathrm{WBC} \geqslant 3.5 \times 10^{9} \mathrm{l}^{-1}\right.$, neutrophils $>$ $1.5 \times 10^{9} 1^{-1}$, platelets $\geqslant 100 \times 10^{9} 1^{-1}$, bilirubi $\leqslant 1.25 \times$ upper limit of normal and serum aspartate aminotransferase, alanine aminotransferase, and alkaline phosphatase $<2.5$ the upper limit of normal, plasma creatinine $\leqslant 130 \mu \mathrm{mol}^{-1}$ or calculated creatinine clearance according to the Cockroft formula $\geqslant 60 \mathrm{ml} \mathrm{min}^{-1}$ ). The study was managed and monitored by the Cancer Research UK Drug Development Office and conducted according to Good Clinical Practice (GCP) at the Royal Marsden Hospital (Sutton) and the Beatson Oncology Centre (Glasgow). The protocol was reviewed by the Cancer Research UK Central Institutional Review Board (CIRB) protocol review committee and the clinical research ethics committees at the above centres. All patients gave written informed consent before entry to the study.

\section{Treatment and dose escalation}

Seliciclib was supplied by Cyclacel Ltd (Dundee, UK) as 50 and $200 \mathrm{mg}$ capsules, stored at room temperature. Capsules were taken twice daily with the patient fasting $2 \mathrm{~h}$ before and after drug dosing. Patients were asked to record daily the number of capsules taken, the time of intake and also when they had last eaten. Compliance was assessed by counting the number of capsules returned by the patient at the end of each treatment cycle. Patients were hospitalised for the first cycle of treatment to facilitate toxicity monitoring and PK sampling. Thereafter, they were treated on an outpatient basis.

The starting dose of seliciclib was selected on the basis of animal toxicology and PK experiments and a single dose study in patients over the dose range $50-200 \mathrm{mg}$. A dose of $400 \mathrm{mg}\left(250 \mathrm{mg} \mathrm{m}^{-2}\right)$ was predicted to achieve meaningful plasma concentrations $(>10 \mu \mathrm{M})$ based on preclinical PK data, and a single-dose human study demonstrated that the elimination half-life was suitable for twice daily administration, although the interindividual variability in clearance was high (De la Motte and Gianella-Borradori, 2004). It was therefore decided to start at $100 \mathrm{mg}$ P.O. twice daily (b.i.d.) to be administered for 7 days every 3 weeks. At least three patients were treated at each dose level. Planned dose increments were at least $100 \%$ until significant drug-related toxicity was observed (grade 1 non-haematological or grade 2 neutropenia or thrombocytopenia), and $40 \%$ thereafter.

Dose-limiting toxicity (DLT) was defined as drug-related toxicity in the first cycle, including grade 4 neutropenia for 7 days or more, neutropenic fever, platelet count $<25 \times 10^{9} 1^{-1}$ and/ or non-haematological toxicity $\geqslant$ grade 3 . This definition excluded nausea and vomiting subsequently responding to antiemetic therapy and reversible grade 3 increases in liver transaminases. The maximum tolerated dose (MTD) was defined as a dose below which DLT occurred in more than $30 \%$ of the patients. Toxicities were evaluated according to the National Cancer Institute Common Toxicity Criteria, version 2.0.

\section{Treatment assessment}

Before commencing treatment, a complete medical history was taken and physical examination was performed. The following pretreatment baseline examinations were performed within 4 
weeks of starting treatment: full blood count, including white blood cell differential; reticulocyte count, haptoglobin, prothrombin time and Coombs test; serum biochemistry, including sodium, potassium, urea, creatinine, calcium, phosphorus, creatine kinase and isoenzymes, total protein, albumin, bilirubin (direct and total), alkaline phosphatase (ALP), alanine aminotransferase (ALT), aspartate aminotransferase (AST), $\gamma$-glutamyl transferase $(\gamma \mathrm{GT})$, lactate dehydrogenase and uric acid; urinalysis; pregnancy test, electrocardiogram and a chest X-ray. Weekly evaluations included history, physical examination, toxicity assessment according to the CTC criteria version 2.0 and full blood count and serum chemistries. Radiographic assessment of tumour was performed within 3 weeks before the patient starting treatment and after every two cycles, according to standard RECIST methodology. Patients were taken off protocol upon disease progression, unacceptable toxicity, investigator discretion, serious violation of protocol or at their own request, and were followed for 28 days after the last administration of the study drug.

\section{Sample collection and pharmacokinetic analysis}

For PK analysis, blood samples (approximately $8 \mathrm{ml}$ each) were collected in tubes with lithium heparin anticoagulant at the following time points: pre-dose and $0.5,1,1.5,2,4,8,12$ (presecond dose), 13, 14 and $24 \mathrm{~h}$ (pre-dose if day 2) after dosing on both days 1 and 7 of the first cycle. The samples were then centrifuged at 3000 r.p.m at $4{ }^{\circ} \mathrm{C}$ for $10 \mathrm{~min}$, the plasma transferred to $2 \mathrm{ml}$ cryovials and frozen at $-70^{\circ} \mathrm{C}$ until analysis. Urine was collected post-dosing for $24 \mathrm{~h}$ on the first day of seliciclib administration, the total urine volume was recorded and an aliquot stored at $-20^{\circ} \mathrm{C}$ for analysis. Concentrations of seliciclib and its principal metabolites in plasma and urine were determined according to a validated liquid chromatography-mass spectrometry detection method at Quintiles, UK and ACC, Germany.

Pharmacokinetic (PK) analysis was performed using the trapezoidal method and log-linear regression. The following parameters were determined either by calculation or observation: the observed maximal concentration $\left(C_{\max }\right)$, the time of observed maximal concentration $\left(T_{\max }\right)$, the area under the concentrationtime curve calculated by the trapezoidal rule (time 0 to last sample with a quantifiable concentration (AUC $(0-\mathrm{tz})$ ) and extrapolated to infinity $\left(\mathrm{AUC}_{(0-\infty)}\right)$, the terminal rate constant, by log-linear regression $\left(\lambda_{\mathrm{z}}\right)$ and the terminal half-life from $\lambda_{\mathrm{z}}$. The value of AUC $_{(0-\infty)}$ was considered unreliable if the terminal area beyond the last quantified sample was greater than $20 \%$ of the total $\mathrm{AUC}_{(0-\infty)}$. Pharmacokinetic (PK) data analysis was carried out using a noncompartmental analysis model with the aid of Kinetica (Innaphase Limited, Amersham, UK).

\section{Sample collection and pharmacodynamic studies}

Preclinical studies indicated that inhibition of RB phosphorylation in tumour could be detected at doses associated with inhibition of tumour growth. It was not clear whether surrogate tissues would provide a similar pharmacodynamic (PD) signal. Samples for PD analysis were taken predose and on days 3, 5, 7 and 14 during the first cycle of treatment with seliciclib. Whole blood was collected from patients at selected time points into lithium-heparin Vacutainer ${ }^{\circledR}$ tubes (Vacutainer, BD, Franklin Lakes, NJ, USA). Each tube was inverted several times to ensure thorough mixing and then mixed with sterile $0.9 \%$ saline at a ratio of $1: 1$. This was then layered over ficol (Lymphoprep ${ }^{\mathbb{R}}$ Nycomed, Oslo, Norway) and centrifuged at 1200 r.p.m. ( $400 \mathrm{~g}$ equivalent) at $18^{\circ} \mathrm{C}$ for $30 \mathrm{~min}$. The resulting mononuclear cell layer was then removed and resuspended in $0.9 \% \mathrm{NaCl}$. Cell pellets were obtained by centrifugation $(600 \mathrm{~g})$ and flash frozen in liquid nitrogen. Mononuclear cells were then stored at $-70^{\circ} \mathrm{C}$ in labelled cryovials until further analysis.
Pharmacodynamic analysis was based on molecular markers developed in preclinical models, including the HCT116 human colon tumour xenograft, namely inhibition of RB and RNA polymerase II phosphorylation and downregulation of cyclin D1 (Whittaker et al, 2004; Raynaud et al, 2005). Cell pellets were treated with lysis buffer $(50 \mathrm{~mm}$ Tris $-\mathrm{HCl}, 150 \mathrm{~mm} \mathrm{NaCl}, \mathrm{pH} 7.5$, $1 \% \mathrm{NP} 40,2 \mathrm{~mm}$ PMSF, $10 \mu \mathrm{g} \mathrm{ml}^{-1}$ aprotinin, $10 \mu \mathrm{g} \mathrm{ml}^{-1}$ leupeptin, $1 \mathrm{~mm}$ activated sodium orthovanadate, $1 \mathrm{~mm}$ sodium fluoride and $10 \mathrm{~mm} \beta$-glycerophosphate) for $30 \mathrm{~min}$ on ice. Samples $(20-100 \mu \mathrm{g}$ of protein) were heat-denatured in Laemmli sample buffer $(10 \%$ glycerol, $5 \% \quad \beta$-mercaptoethanol, $2 \%$ sodium dodecyl sulphate (SDS), $62.5 \mathrm{~mm}$ Tris ( $\mathrm{pH}$ 6.8), $0.05 \%$ bromphenol blue, final concentrations) and separated by SDS-polyacrylamide gel electrophoresis (PAGE) using pre-cast 10 or 15 -well 20 or $10 \%$ Tris-glycine polyacrylamide gels (Invitrogen, Netherlands). All constituents of lysis and loading buffers were obtained from Sigma (Poole, UK) The membrane was then blocked with $0.5 \%$ casein blocking buffer $(0.5 \%$ casein hammerstein, $154 \mathrm{~mm} \mathrm{NaCl}, 10 \mathrm{~mm}$ Tris base, $\mathrm{pH}$ 7.6) incubated overnight with primary antibody (phospho-RB Ser-780: Cell Signalling Technology, MA, USA); total RB protein, sc-50, (Santa Cruz Biotechnology, CA, USA); cyclin A, Ab 6 (Neomarkers, Fremont, CA, USA); Phospho ser5 pol II, R2033-20, US Biological (Swampscott, MA, USA); Phospho ser2 pol II, H5 MMS-129R (Covance, Berkeley, CA, USA); total pol II, Ab5408 (Abcam, Cambridge, UK); and GAPDH (Chemicon, CA, USA).

Visualisation of the bound primary antibody was performed by probing with horseradish peroxide (HRP)-conjugated secondary antibody (anti-rabbit (Bio-Rad, CA, USA); anti-mouse (Amersham Biosciences, Bucks, UK)). The membrane was immersed in ECL reagent (Pierce Biotechnology, Rockford, IL, USA) for $1 \mathrm{~min}$ and then exposed to photographic film (Hyperfilm, Amersham Biosciences, Bucks, UK) and processed.

\section{RESULTS}

\section{General}

Between August 2001 and September 2003, 22 patients with metastatic disease were enrolled from the two centres and their demographic characteristics are listed in Table 1 . All 22 patients were eligible, but one was withdrawn before receiving treatment owing to rapid disease and symptom progression. Patients had received a median number of two prior chemotherapy regimens. A total number of 42 fully assessable courses were administered. The median number of courses per patient was 2 ; range $1-6$. The following dose levels were studied: $100 \mathrm{mg}$ b.i.d, $200 \mathrm{mg}$ b.i.d and $800 \mathrm{mg}$ b.i.d.

No significant toxicity was seen at the $100 \mathrm{mg}$ dose level at which three patients were treated. At the $200 \mathrm{mg}$ dose level, one patient with advanced colorectal cancer experienced an increase in plasma and liver enzymes, consistent with cholestasis, which did not revert to baseline during the period of the study. The patient's liver biochemistry at baseline was within normal limits but, between days 21 and 28 grade 3 elevations of bilirubin, $\gamma \mathrm{GT}$, ALP, AST and ALT were noted. Subsequent ultrasound investigation revealed new liver metastases, but no features of biliary obstruction. The changes in liver biochemistry persisted and did not revert to baseline upon cessation of treatment, suggesting this may have been tumour-related. The patient was consequently withdrawn from the trial. Nonetheless, this was classified as a DLT, as it was not transient. Three additional patients were recruited at this dose level and no further DLT was reported. At this point, review of the PK data obtained at the 100 and $200 \mathrm{mg}$ dose levels indicated that these doses resulted in very low drug exposure. In addition, $400 \mathrm{mg}$ twice daily for 5 days every 3 weeks appeared to be well tolerated in another seliciclib Phase I trial ongoing at the time. A protocol 
Table I Patient characteristics

\begin{tabular}{|c|c|}
\hline \multicolumn{2}{|l|}{ Gender } \\
\hline Male & 15 \\
\hline Female & 6 \\
\hline Median Age (range) years & $62(39-73)$ \\
\hline \multicolumn{2}{|l|}{ Performance status } \\
\hline 0 & 2 \\
\hline 1 & 17 \\
\hline 2 & 2 \\
\hline \multicolumn{2}{|l|}{ Site of primary disease } \\
\hline Colorectal & 9 \\
\hline Ovarian & 2 \\
\hline Unknown primary & 2 \\
\hline Lung & I \\
\hline Cervical & । \\
\hline Prostate & I \\
\hline Other & 5 \\
\hline \multicolumn{2}{|l|}{ Prior treatment regimens } \\
\hline \multicolumn{2}{|l|}{ Chemotherapy } \\
\hline l & 6 \\
\hline 2 & 9 \\
\hline 3 & 7 \\
\hline $3+$ & I \\
\hline Prior hormone therapy & I \\
\hline Prior radiation & 8 \\
\hline No. of cycles of seliciclib administered & 42 \\
\hline Median per patient & 2 \\
\hline Range & $(1-6)$ \\
\hline
\end{tabular}

amendment was made to dose escalate from 200 to $800 \mathrm{mg}$. At the $800 \mathrm{mg}$ dose level, reversible grade 1 and 2 increases in plasma creatinine, reversible grade 3 and 4 hypokalaemia, reversible grade 3 hyponatraemia and a grade 3 skin rash were observed, so the cohort was expanded to six patients. No further DLTs were experienced in the additional group of three patients. A further protocol amendment allowed a cohort expansion of an additional group of six patients (12 patients in total) to facilitate closer investigation of the putative renal/biochemical toxicities of seliciclib. Four of the final six patients treated at $800 \mathrm{mg}$ experienced seliciclib-related DLTs. In three of these patients, this involved reversible elevations of $\gamma \mathrm{GT}$, and in one patient, hyperglycaemia and glycosuria.

\section{Toxicity profile}

Overall haematological toxicity was mild and did not result in DLT. Nausea was the most commonly reported adverse event and increased in frequency with increasing dose. At the $800 \mathrm{mg}$ dose level, 11 of the 12 patients experienced nausea, although this was not dose-limiting. This symptom was satisfactorily managed with routine antiemetics, including $5-\mathrm{HT}_{3}$ antagonists. Other commonly reported drug-related adverse events included lethargy, fatigue and anorexia. Hypokalaemia, rash and fatigue were the principal DLTs, and were mainly observed at the $800 \mathrm{mg}$ b.i.d. dose level. Further details of these adverse events are summarised in Table 2.

\section{Electrolyte disturbance and renal impairment}

Hypokalaemia considered related to seliciclib was noted in patients receiving $800 \mathrm{mg}$ b.i.d. At this dose, hypokalaemia was observed in five of 12 patients $\left(<3.0 \mathrm{mmol}^{-1}\right)$, which was grade 1 in two patients, grade 3 in two patients and grade 4 in one patient. This was not deemed to be a result of nausea and vomiting or concomitant diuretic or nephrotoxic therapy. These biochemical
Table 2 Reported toxicity profile during the first course of seliciclib treatment

\begin{tabular}{lccc}
\hline & $\begin{array}{c}\text { Seliciclib } \\
\mathbf{1 0 0} \mathbf{~ m g ~ b . i . d}\end{array}$ & $\begin{array}{c}\text { Seliciclib } \\
\mathbf{2 0 0} \mathbf{~ m g} \text { b.i.d }\end{array}$ & $\begin{array}{c}\text { Seliciclib } \\
\mathbf{8 0 0 ~} \mathbf{~ m g} \text { b.i.d }\end{array}$ \\
\hline No. of subjects & 3 & 6 & 12 \\
No. with grade 3 \& & 0 & 2 & 7 \\
adverse events & & & \\
$\gamma$ GT increase & 0 & 1 & 3 \\
Hypokalemia & 0 & 0 & 3 \\
Hyperglycaemia & 0 & 0 & 1 \\
Hyponatraemia & 0 & 0 & 1 \\
Rash & 0 & 0 & 1 \\
Hypotension & 0 & 0 & 1 \\
Lymphopenia & 0 & 0 & 1 \\
Anorexia & 0 & 0 & 1 \\
Fatigue & 0 & 1 & 1 \\
ALT increased & 0 & 1 & 0 \\
AST increased & 0 & 1 & 0 \\
Alk Phos increase & 0 & 1 & 0 \\
Bilirubin increase & 0 & 1 & 0 \\
CKMB increase & 0 & & \\
\hline
\end{tabular}

changes were first observed on day 7 of seliciclib dosing, as routine electrolyte testing was initially performed only on days 1 and 7 during the treatment period. Additional blood testing revealed that these changes occurred as early as day 3 of treatment. Hypokalaemia was easily reversible with potassium supplementation and was not associated with any electrocardiographic changes. Clinically significant elevations in urea and creatinine were also observed and were correctable with increased oral or intravenous fluids. These biochemical changes appeared to occur separately from the aforementioned hypokalaemia.

This toxicity can be characterised further by describing a typical patient history. In one patient treated at $800 \mathrm{mg}$ b.i.d., serum potassium fell to $2.2 \mathrm{mmoll}^{-1}$ on day 7 of course 1 , with a creatinine level just above normal and no other identifiable cause of hypokalaemia. This electrolyte disturbance required hospitalisation, for administration of intravenous and oral potassium supplementation. This patient also developed hypokalaemia after receiving course 2 , with a nadir of $2.5 \mathrm{mmoll}^{-1}$, and on this occasion associated with hyponatraemia $\left(127 \mathrm{mmoll}^{-1}\right)$ and hypotension $(82 / 52 \mathrm{mmHg})$ with a creatinine that was just above the normal range $\left(130 \mu \mathrm{moll}^{-1}\right.$; normal range $\left.<110 \mu \mathrm{moll}^{-1}\right)$. These were, however, possibly associated with concurrent poor oral fluid intake.

At this point, the $800 \mathrm{mg}$ cohort was expanded further to investigate the possible renal effects of seliciclib. More restrictive eligibility criteria were introduced, including a glomerular filtration rate above $60 \mathrm{ml} \mathrm{min}^{-1}$ (estimated by ${ }^{51} \mathrm{Cr}$-EDTA) clearance or 24-h urine collection for creatinine clearance) and serum potassium within normal limits. In addition, more intensive blood chemistry testing was performed on days $1,3,5$ and 7 . Furthermore, 24-h urine collections were obtained on days 1 and 7 and analysed for creatinine clearance, urinary electrolytes and urine microscopy. No overall changes were seen in urinalysis, but in one patient with hypokalaemia in this cohort, potassium excretion in the urine was increased on day 7 compared with day 1 , suggestive of renal potassium loss.

In an attempt to understand further the pathogenesis of these biochemical toxicities, three patients were studied more intensively. Urinary retinol-binding protein (RBP), a marker of renal tubular damage was measured in conjunction with functional renal imaging (MAG3 renograms) to estimate renal blood flow pretreatment on days 3 and 14 . One patient experienced a rapid, but reversible, increase in creatinine associated with a reduction in renal perfusion bilaterally, determined by MAG3 perfusion indices. 
This was associated with bilateral increases in renal parenchymal retention on day 3 on MAG3 scans, which reverted to normal by day 14 (see Figure 1). By contrast, the other two patients experienced no decline in renal function and showed no change in MAG3 renograms. There were no consistent changes in urinary RBP in any of these patients (Table 3 ).

\section{Hyperglycaemia}

Seliciclib-induced hyperglycaemia was observed in five patients at $800 \mathrm{mg}$ b.i.d., but reached grade 3 in only one patient with associated glycosuria. This patient was not receiving concurrent corticosteroids. The hyperglycaemia was transitory, being noted by the end of the treatment period on day 7 and fully normalised on cessation of drug therapy.

\section{Other non-haematological toxicity}

One patient at the $800 \mathrm{mg}$ dose level experienced a grade 3 rash occurring on day 7 of the first treatment cycle. This was characterised by an intensely pruritic and erythematous, urticarial rash of diffuse distribution affecting the trunk, lower limbs and arms associated with mild systemic upset and pyrexia. The patient was treated with topical steroid creams, emollients and antihistamines, and the rash resolved. The patient was subsequently rechallenged with a second cycle of treatment and within $4 \mathrm{~h}$ of the first seliciclib dose, the rash reappeared. The patient was commenced on oral steroids and the rash again resolved within 2 weeks. Subsequent expert dermatological review confirmed that these episodes were likely to be drug related, and were of a vasculitic appearance. Associated biochemical abnormalities in the same patient were also noted on day 7 of the treatment period during both cycles 1 and 2, namely hypokalaemia, raised urea and
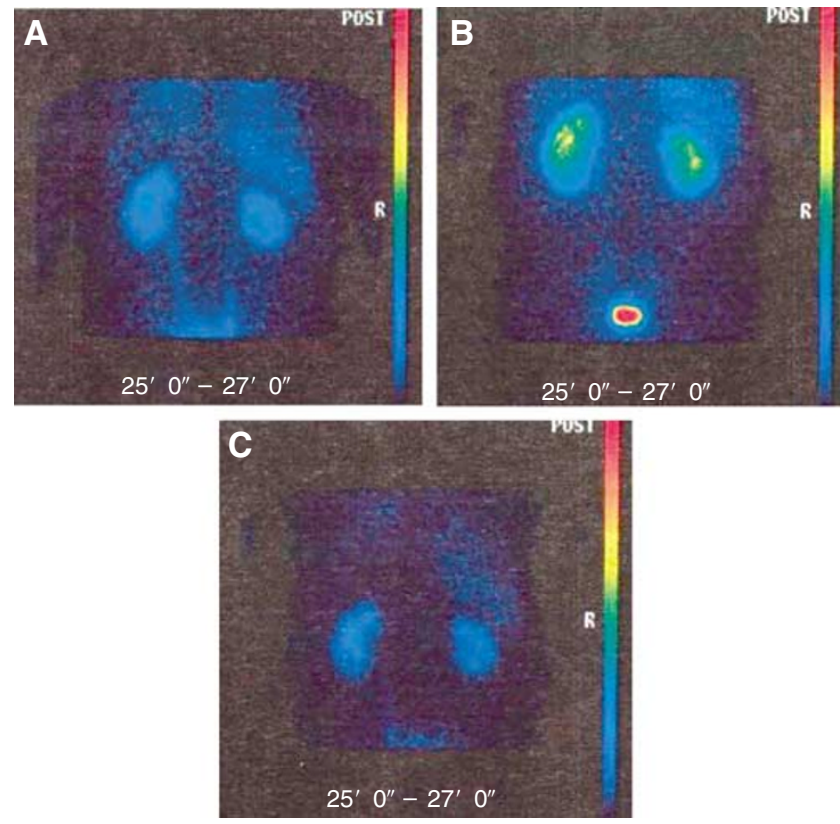

Figure I Functional renal perfusion imaging by MAG3 nuclear scanning, performed pretreatment $(\mathbf{A})$ and post-treatment on days $3(\mathbf{B})$ and 14 (C), in a patient who experienced a reversible decline in renal function when treated with seliciclib at $1600 \mathrm{mg} \mathrm{day}^{-1}$. Baseline pre-treatment renogram (A) shows slight asymmetry in renal function with the right kidney (dark green) slightly poorer than the left (light green). Day 3 renogram (B) shows changes consistent with bilateral parenchymal retention and delayed intrarenal transit bilaterally, which then almost completely recovers by day 14 (C).
Table 3 Urinary retinol binding protein levels following treatment with seliciclib

\begin{tabular}{lccc}
\hline & \multicolumn{3}{c}{$\begin{array}{c}\text { Urinary retinol binding protein } \\
(\mathbf{0 . 1 0 - \mathbf { 1 0 0 . 0 }} \mathbf{~ m g / m o l ~ c r e a t i n i n e ) ~}\end{array}$} \\
\cline { 2 - 4 } & Patient $\mathbf{2 0}$ & Patient 2 I & Patient 22 \\
\hline Pretreatment & 8.7 & 136.4 & 6.8 \\
Day 3 & 59.2 & 44.7 & 7.9 \\
Day 14 & 20.8 & 25.7 & 40.1 \\
\hline
\end{tabular}
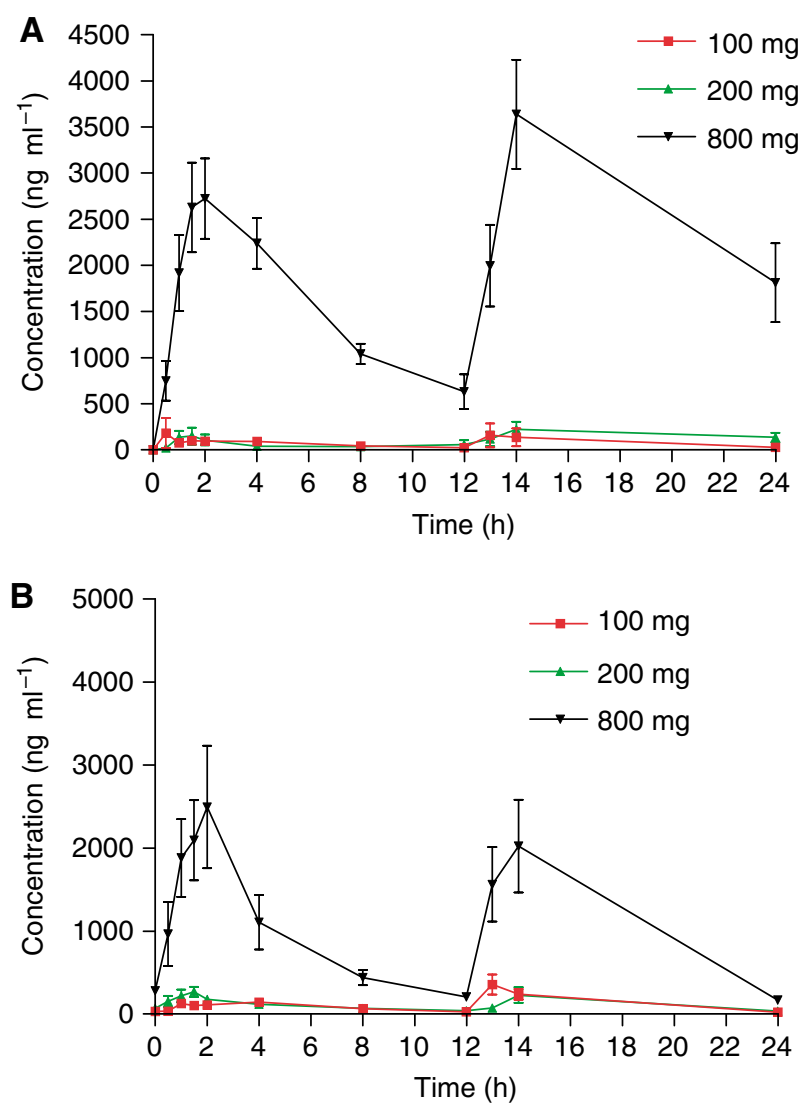

Figure 2 Concentration-time pharmacokinetic profiles (mean \pm standard deviation) for all the evaluated dose levels of seliciclib (I00, 200, $800 \mathrm{mg}$ ) on both days I (A) and 7 (B).

creatinine and hyperglycaemia. These values were within normal limits before treatment and resolved within days of finishing seliciclib.

Derangements in liver biochemistry, principally, elevations of $\gamma \mathrm{GT}$ and ALP were also reported during the 7-day treatment period at both $200 \mathrm{mg}$ b.i.d. (4 out of 6 patients) and $800 \mathrm{mg}$ b.i.d. (10 out of 12) dose levels. Most of the derangement was mild to moderate. Raised liver biochemistry tests were seen by day 7 and were reversible at the end of the course with the exception of the DLT at the $200 \mathrm{mg}$ dose level, which was probably tumour related.

\section{Pharmacokinetics}

The mean concentration vs time profile for each dose level is shown in Figure 2. Seliciclib was absorbed relatively slowly with a mean $T_{\max }$ of $2.55 \mathrm{~h}$ (range $1-4 \mathrm{~h}$ ). The plasma clearance was greater than liver blood flow and the terminal half-life was similar in all dose groups $(2-5 \mathrm{~h})$. Table 4 summarises the pharmaco- 
Table 4 Summary of Seliciclib pharmacokinetics during course I

\begin{tabular}{|c|c|c|c|c|c|c|c|}
\hline & \multicolumn{2}{|c|}{ Dose } & $\frac{C_{\max }\left(\mathbf{n g ~ m} \mathbf{~ I}^{-\mathbf{1}}\right)}{248}$ & $\frac{T_{\max }(\min )}{112}$ & $\frac{\boldsymbol{t}_{\mathbf{1 / 2}}(\mathbf{m i n})}{267}$ & 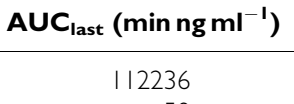 & 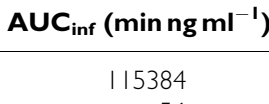 \\
\hline & & $\% C V$ & 91 & 101 & 55 & 58 & 56 \\
\hline & & $\% \mathrm{CV}$ & 123 & 95 & 42 & 62 & 59 \\
\hline & & Median & 80 & 167 & 149 & 185025 & 230597 \\
\hline & $800 \mathrm{mg}(n=12)$ & Mean & 3197 & 177 & 219 & 3163604 & 3177921 \\
\hline \multirow[t]{6}{*}{ Day 7} & $100 \mathrm{mg}(n=3)$ & Mean & 162 & 190 & 268 & 171555 & 173422 \\
\hline & & $\% C V$ & 15 & 46 & 24 & 36 & 35 \\
\hline & & Median & 152 & 240 & 234 & 181815 & 182620 \\
\hline & $200 \mathrm{mg}(n=6)$ & Mean & 310 & 62 & 781 & 175903 & 255297 \\
\hline & & $\% C V$ & 43 & 71 & 173 & 59 & 89 \\
\hline & & Median & 303 & 60 & 228 & 184620 & 208748 \\
\hline
\end{tabular}
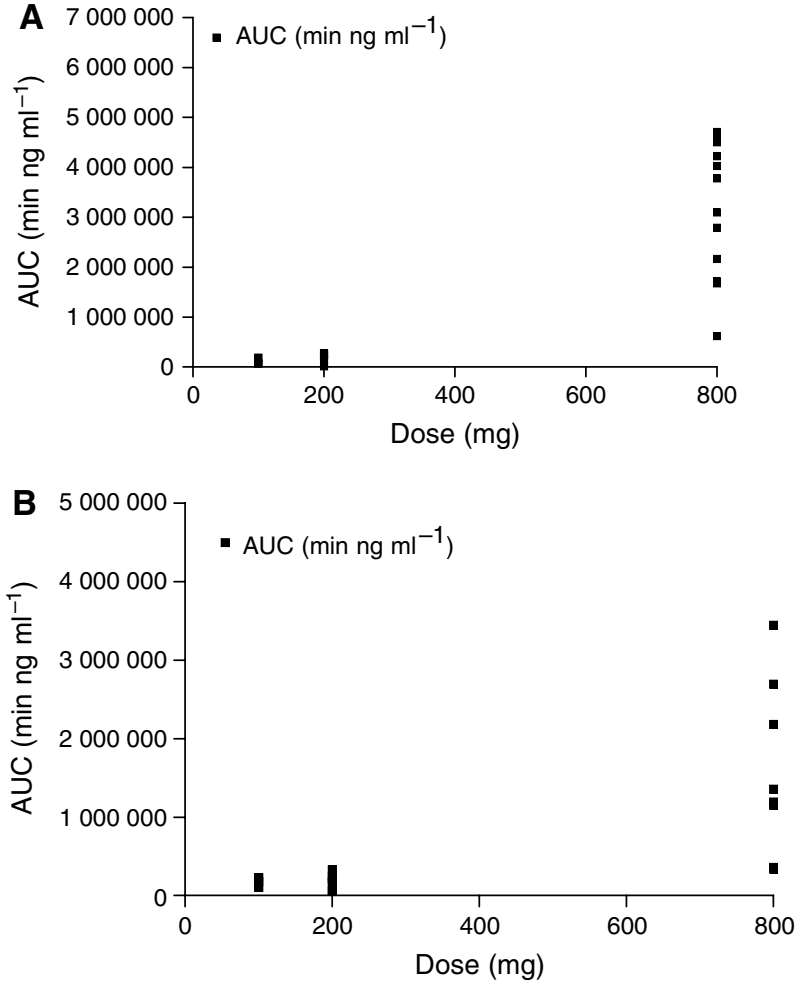

Figure 3 Seliciclib $A \cup C_{(\text {last })}$ values for all tested dose levels, on both days $I(\mathbf{A})$ and 7 (B), as a function of daily administered dose.

kinetic parameters for patients treated with seliciclib at each dose level. Area-under-the-curve (AUC) and $C_{\max }$ of seliciclib increased with dose (Figures 3 and 4). There is a suggestion of nonlinearity, but this is unclear from the limited data generated by this study. There were no significant differences in the $\mathrm{PK}$ parameters calculated on days 1 and 7. The ratio of the carboxylic acid derivative which is the major metabolite (De la Motte and GianellaBorradori, 2004; Nutley et al, 2005) to the parent compound, varied from 0.8 to 43 (data not shown). However, there was no
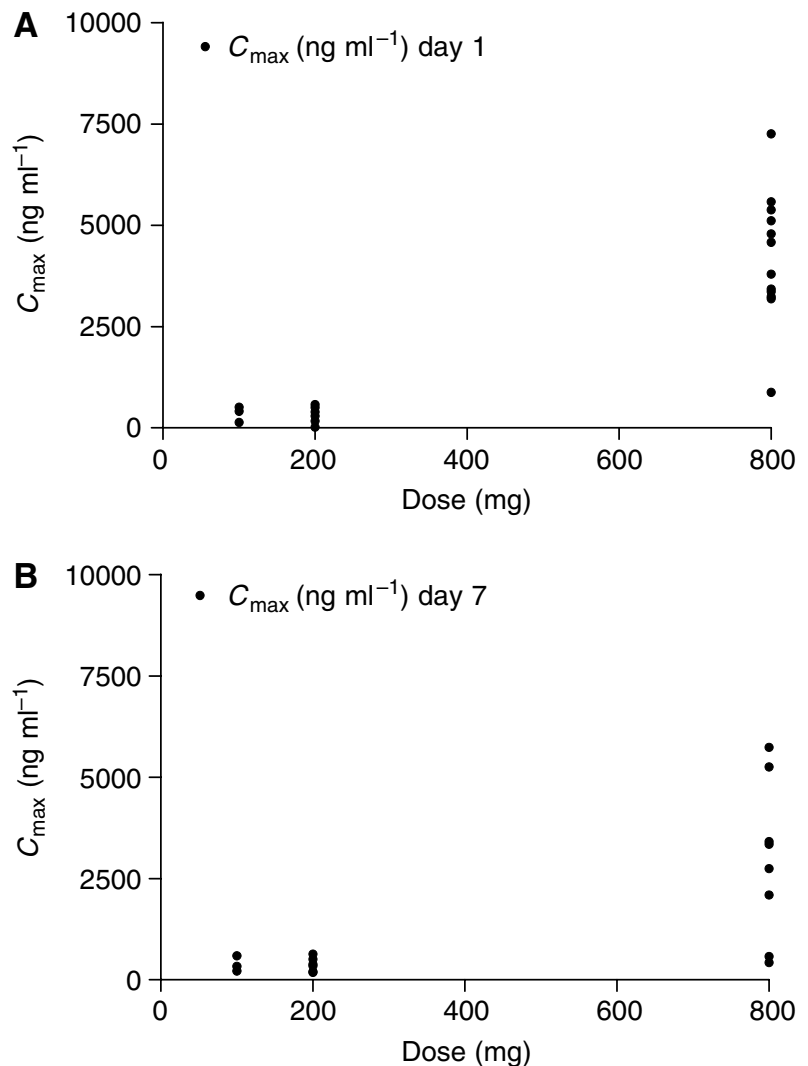

Figure 4 Seliciclib $C_{\max }$ values for all tested dose levels, on both days I (A) and 7 (B), as a function of daily administered dose.

correlation between the ratio of metabolite to parent and the plasma clearance of the parent drug. The mean $C_{\max }$ at the $800 \mathrm{mg}$ dose level $\left(3 \mathrm{mg} \mathrm{ml}^{-1}, 10 \mu \mathrm{M}\right)$ was in the range of $\mathrm{IC}_{50}$ values reported for seliciclib in vitro activity (McClue et al, 2002; Nutley et al, 2005; Raynaud et al, 2005), but this concentration was not sustained for the period required to exert an antitumour effect in vitro. 


\section{Pharmacodynamics}

Retinoblastoma phosphorylation and cyclin D1 levels were studied in peripheral blood mononuclear cells, compared with appropriate controls. No reliably detectable alterations were observed. Tumour biopsies were not obtained in this study.

\section{Antitumour activity}

No objective tumour responses were observed. One patient with metastatic ovarian cancer, whose disease was progressing before treatment with seliciclib, had disease stabilisation for a total of six courses, with no change in her CA-125 tumour marker. Two of three patients at the $100 \mathrm{mg}$ b.i.d. dose level, two of six at the $200 \mathrm{mg}$ b.i.d. dose level and three of 12 patients at the $800 \mathrm{mg}$ b.i.d. dose level had stable disease after course 2, but progressed by course 4

\section{DISCUSSION}

Cell-cycle dysregulation is a hallmark of malignancy. The Cdks play a crucial role in controlling progression through the cell cycle, and genetic and epigenetic mechanisms frequently result in their deranged expression and/or activity in oncogenesis. The development of Cdk inhibition has therefore been pursued as a potential therapeutic strategy, although the development of selective inhibitors of this serine-threonine kinase family has been particularly challenging in view of the high degrees of sequence homology between Cdks and other kinases. It has been reported that Cdk inhibitors could inhibit tumour cell growth in preclinical models, but many questions remain about which Cdks should be targeted for anticancer therapy. These concerns have been fuelled by RNA interference and knockout mice studies, indicating that functional redundancy may exist between different Cdks, with the loss of Cdk2 alone failing to inhibit tumour cell proliferation (Ortega et al, 2003; Tetsu and McCormick, 2003), and the loss of both Cdk4 and Cdk6 not preventing cell cycling (Malumbres et al, 2004). These data suggest that the optimal pharmacologic Cdk inhibitor should selectively inhibit a broad spectrum of Cdks, including Cdk1, 2, 4, 6, yet spare other kinases whose inhibition results in nonspecific toxicity (Sausville, 2002).

Seliciclib is an orally bioavailable selective inhibitor of Cdk 1, 2, 7 and 9 (McClue et al, 2002; Whittaker et al, 2004). Cassette dosing PK studies indicated that seliciclib had the most favourable PK profile of a library of 107 different 2,6,9-tri-substituted purine Cdk2 inhibitors, prepared by parallel synthesis (Raynaud et al, 2004). Seliciclib showed promising activity in preclinical models, and decreased both RB and RNA Pol II phosphorylation and total RNA Pol II protein, suggesting that in addition to Cdk2 inhibition, it also inhibits transcription, possibly via inhibition of Cdk7 and Cdk9 (McClue et al, 2002; Whittaker et al, 2004; MacCallum et al, 2005; Raynaud et al, 2005). Similarly, seliciclib also decreases cyclin D1 protein levels independently of the p38SAPK and phosphatidylinositol 3-kinase pathways and causes a reduction in the expression of cyclins D1, A and B1, presumably by the same mechanism. Its cell-cycle effects include a reduction of cells in $G_{1}$, inhibition of bromodeoxyuridine incorporation during $S$ phase and a moderate increase in $\mathrm{G}_{2} / \mathrm{M}$ phase.

We now report the clinical evaluation of the orally bioavailable selective Cdk inhibitor seleciclib in cancer patients, using oral dosing b.i.d. for 7 days every 3 weeks. Preclinical toxicology studies with 28 days treatment in rodents reported anaemia, leucocytosis, bone marrow hyperplasia, polydipsia, polyuria and gastric ulceration. Preclinical data indicated that exposure to seliciclib in the concentration range 7.9-30.2 $\mu \mathrm{M}$ over a $24-\mathrm{h}$ period could achieve clinically relevant biological effects (De la Motte and Gianella-Borradori, 2004; Whittaker et al, 2004; Tang et al, 2005). In vivo studies also indicated that a single dose of
$500 \mathrm{mg} \mathrm{kg}^{-1}$ (2750 $\mathrm{mg} \mathrm{m}^{-2}$ of mouse body surface area) achieved levels of $\geq 10 \mu \mathrm{m}$ for $24 \mathrm{~h}$ (Raynaud et al, 2005). In men, a single dose of $250 \mathrm{mg} \mathrm{m}^{-2}$ ( $400 \mathrm{mg}$ dose for a patient with a body surface area of $1.8 \mathrm{~m}^{2}$ ) was predicted to achieve the same level for $4 \mathrm{~h}$. However, in a single dose bioavailability study in healthy volunteers, considerable interindividual variability in drug exposure was observed, with most of the drug being cleared by $12 \mathrm{~h}$ (De la Motte and Gianella-Borradori, 2004).

In this phase I trial, no significant drug-related toxicity was observed at the 100 or $200 \mathrm{mg}$ b.i.d. dose levels, and PK data suggested that these doses resulted in low drug exposure (see Figure 3), hence the dose was increased to $800 \mathrm{mg}$ b.i.d. for 7 days. At this level, dose-limiting toxicities were reported comprising reversible hypokalaemia, hyponatraemia, elevated $\gamma \mathrm{GT}$, hyperglycaemia and a generalised vasculitic skin rash. In addition, a rapid rise in creatinine during the administration period was also frequently observed. Although this did not reach grade 3 and appeared to be reversible on stopping the drug, it was unexpected and of concern in the absence of a clear explanation. Similar toxicities have been reported in a separate phase I study utilising a 5-day, twice-daily administration, oral schedule (Pierga et al, 2003). In that study, vomiting, skin rash, hypokalaemia and raised creatinine were also seen. The recommended phase II dose in that study was $2500 \mathrm{mg}$ per day for 5 days, although this was reported to be associated with manageable grade 3 hypokalaemia and grade 3 skin rash. Preclinical toxicology had reported seliciclib-related polyuria and polydypsia, but not renal dysfunction or hypokalaemia.

The pathogenesis of the creatinine rise is not fully understood, but may be associated with a reversible reduction in renal blood flow. Some evidence for this was obtained from the serial evaluation of MAG3 isotope renograms that detected a significant, reversible, decrease in renal blood flow in one patient, following seliciclib therapy. The pathogenesis of this possible alteration in renal blood flow remains unexplained. It has been proposed that binding of seliciclib to unrelated targets such as adenosine receptors, which regulate renal blood flow, could explain these findings, but this has not been confirmed to date (Benson et al, 2005). The reversibility of the renal dysfunction and absence of changes in urinary retinol-binding protein do, however, suggest that this was not related to clinically significant tubular damage.

Although clearly distinct from the renal dysfunction, in that the two events did not always occur simultaneously, reversible doselimiting hypokalemia was also observed during this study. Whereas this was noted at the $200 \mathrm{mg}$ dose level, potassium levels below $3.0 \mathrm{mmoll}^{-1}$ were only observed at $800 \mathrm{mg}$, with potassium levels as low as $2.2 \mathrm{mmoll}^{-1}$ in one patient. The hypokalaemia was easily and rapidly reversible with potassium supplementation and on discontinuation of seliciclib dosing. It was, however, thought to be potentially dangerous and would warrant very close monitoring. The pathogenesis of the hypokalaemia has not been elucidated. As renal collecting duct and tubular reabsorption of potassium (involving carbonic anhydrase) is critical to potassium homeostasis, these processes may be implicated. Further studies on the effects of seliciclib on adenosine receptors, carbonic anhydrase and renal collecting duct and tubular function may be useful.

The pharmacokinetic data indicate that seliciclib levels were not maintained at the level associated with antitumour activity in some xenograft models. The mean maximum plasma concentration achieved at the highest dose level was approximately $3 \mu \mathrm{g} \mathrm{ml}^{-1}$ $(10 \mu \mathrm{M})$, whereas in the mouse, a peak seliciclib concentration of approximately $100 \mu \mathrm{M}$ and nearly $24 \mathrm{~h}$ of exposure to $>10 \mu \mathrm{M}$ was achieved with a single dose of seliciclib at $500 \mathrm{mg} \mathrm{kg}^{-1}$ p.o. When administered b.i.d., this resulted in significant growth delay of a human tumour xenograft (Raynaud et al, 2005). However, the lowest effective dose in this xenograft model was not established. More sensitive tumours may not require such high drug levels and it is unclear whether this discrepancy in drug exposure was responsible for the failure to demonstrate an effect on $\mathrm{RB}$ 
phosphorylation and cyclin D1 in peripheral blood mononuclear cells. A number of other factors may have contributed; for example, peripheral blood mononuclear cells may be inappropriate as a surrogate tissue for these agents in man, as they are not actively dividing. Buccal mucosa scrapes have been used to demonstrate changes in $\mathrm{p} 53$ and phospho-RB in response to flavopiridol, although this did not translate into detectable changes in tumour biopsies (Tan et al, 2004). In a separate study with flavopiridol, flow cytometric studies to examine cell cycle changes and apoptosis in peripheral blood mononuclear cells were uninformative (Thomas et al, 2002).

Side effects such as neutropenia and gastrointestinal toxicity, such as have been reported with other Cdk inhibitors, were not observed during this study. It is not entirely clear what side effects are to be expected from a broad-spectrum Cdk inhibitor, such as seliciclib. If the biologically important effects of seliciclib are on transcriptional regulation, it is hard to predict exactly what pattern of side effects is likely to be observed with this agent. The observation of seliciclib-related hyperglycaemia in five of 12 patients at the $800 \mathrm{mg}$ dose level is interesting. This has been reported with other Cdk inhibitors, possibly through inhibition of Cdk5, which has a role in regulating insulin secretion (Sausville, 2002).

In this study DLT was observed at $800 \mathrm{mg}$, given b.i.d. for 7 days every 21 days. Although the toxicities were reversible, they were nevertheless of concern and it proved difficult to continue dosing for 7 days in some patients in the presence of a consistently rising creatinine. Plasma concentrations were not maintained above the average $\mathrm{IC}_{50}$ concentration at this dose and schedule. However, shorter duration regimens, that is, b.i.d. administration for 5 days, have proved to be feasible and permit administration of higher daily doses (Pierga et al, 2003). It was the decision of the sponsor to pursue clinical development of the shorter duration regimen and not to continue further investigation of the 7-day continuous dosing regimen. Further investigations are required to explain fully the renal side effects observed with seliciclib. An orally bioavailable Cdk inhibitor such as seliciclib has significant attractions. The development of alternative PD biomarkers will assist the identification of the optimum dose and schedule.

\section{ACKNOWLEDGEMENTS}

We are indebted to the patients and their families, who participated in this clinical trial, and the staff of the Royal Marsden Hospital and the Beatson Oncology Centre, as well as Dr Gary Cook, who interpreted the MAG3 isotope renograms. This work was supported by Cancer Research UK (CUK) programme grant number C309/A2187. Paul Workman is a Cancer Research UK Life Fellow.

Prior presentation: The American Society of Clinical Oncology in 2003.

\section{REFERENCES}

Alvi AJ, Austen B, Weston VJ, Fegan C, MacCallum D, Gianella-Borradori A, Lane DP, Hubank M, Powell JE, Wei W, Taylor AMR, Moss PAH, Tatjana Stankovic T (2005) A novel Cdk inhibitor, CYC202 (R-roscovitine), overcomes the defect in p53-dependent apoptosis in B-CLL by down-regulation of genes involved in transcription regulation and survival. Blood 105: 4484-4491

Bach S, Knockaert M, Reinhardt J, Lozach O, Schmitt S, Baratte B, Koken M, Coburn SP, Tang L, Jiang T, Liang DC, Galons H, Dierick JF, Pinna LA, Meggio F, Tozke F, Schachtele C, Lerman AS, Carno A, Wan Y, Gray N, Meijer L (2005) Roscovitine targets: protein kinase and pyridoxal kinase. J Biol Chem 280: 31208 -31219

Benson B, Kaye S, Workman P, Garrett M, Walton M, de Bono J (2005) Clinical anticancer drug development: targeting the cyclin dependent kinase. Br J Cancer 92: 7-12

De Azevedo WF, Leclerc S, Meijer L, Havlicek L, Strnad M, Kim SH (1997) Inhibition of cyclin-dependent kinases by purine analogues: crystal structure of human cdk2 complexed with roscovitine. Eur J Biochem 243: $518-526$

De la Motte S, Gianella-Borradori A (2004) Pharmacokinetic model of R-roscovitine and its metabolite in healthy male subjects. Int J Clin Pharmacol Ther 42: 232-239

Gray NS, Wodicka L, Thunnissen AM, Norman TC, Kwon S, Espinoza FH, Morgan DO, Barnes G, LeClerc S, Meijer L, Kim SH, Lockhart DJ, Schultz PG (1998) Exploiting chemical libraries, structure, and genomics in the search for kinase inhibitors. Science 281: $533-538$

$\mathrm{Lu} \mathrm{W}$, Chen L, Peng Y, Chen J (2001) Activation of p53 by roscovitinemediated suppression of MDM2 expression. Oncogene 20: 3206-3216

MacCallum DE, Melville J, Frame S, Watt K, Anderson S, GianellaBorradori A, Lane DP, Green SR (2005) Seliciclib (CYC202, RRoscovitine) induces cell death in multiple myeloma cells by inhibition of RNA polymerase II-dependent transcription and down-regulation of Mcl-1. Cancer Res 65: 5399-5407

Malumbres M, Barbacid M (2001) To cycle or not to cycle: a critical decision in cancer. Nat Rev Cancer 1: 222-231

Malumbres M, Sotillo R, Santamaria D, Galan J, Cerezo A, Ortega S, Dubus P, Barbacid M (2004) Mammalian cells cycle without the D-type cyclindependent kinases Cdk4 and Cdk6. Cell 118: 493-504

McClue SJ, Blake D, Clarke R, Cowan A, Cummings L, Fischer PM, MacKenzie M, Melville J, Stewart K, Wang S, Zhelev N, Zheleva D, Lane DP (2002) In vitro and in vivo antitumor properties of the cyclin dependent kinase inhibitor CYC202 (R-roscovitine). Int J Cancer 102: 463-468

Meijer L, Borgne A, Mulner O, Chong JP, Blow JJ, Inagaki N, Inagaki M, Delcros JG, Moulinoux JP (1997) Biochemical and cellular effects of roscovitine, a potent and selective inhibitor of the cyclin-dependent kinases cdc2, cdk2 and cdk5. Eur J Biochem 243: 527-536

Meijer L, Raymond E (2003) Roscovitine and other purines as kinase inhibitors. From starfish oocytes to clinical trials. Acc Chem Res 36: 417-425

Nutley BP, Raynaud FI, Wilson SC, Fischer PM, Hayes A, Goddard PM, McClue SJ, Jarman M, Lane DP, Workman P (2005) Metabolism and pharmacokinetics of the cyclin-dependent kinase inhibitor R-Roscovitine in the mouse. Mol Cancer Ther 4: 125-139

Ortega S, Prieto I, Odajima J, Martin A, Dubus P, Sotillo R, Barbero JL, Malumbres M, Barbacid M (2003) Cyclin-dependent kinase 2 is essential for meiosis but not for mitotic cell division in mice. Nat Genet 35: 25-31

Pierga J, Faivre S, Vera K, Laurence V, Delbaldo C, Bekradda M, Armand JP, Gianella-Borradori A, Dieras V, Raymond E (2003) A phase I and pharmacokinetic (PK) trial of CYC202, a novel oral cyclin-dependent kinase (CDK) inhibitor, in patients (pts) with advanced solid tumors. Proc Am Soc Clin Oncol 22: Abstr. 210

Raje N, Kumar S, Hideshima T, Roccaro A, Ishitsuka K, Yasui H, Shiraishi N, Chauhan D, Munshi NC, Green SR, Anderson KC (2005) Seliciclib (CYC202 or R-roscovitine), a small-molecule cyclin-dependent kinase inhibitor, mediates activity via down-regulation of Mcl-1 in multiple myeloma. Blood 106: $1042-1047$

Raynaud FI, Fischer PM, Nutley BP, Goddard PM, Lane DP, Workman P (2004) Cassette dosing pharmacokinetics of a library of 2, 6, 9trisubstituted purine cyclin-dependent kinase 2 inhibitors by parallel synthesis. Mol Cancer Ther 3: 353-362

Raynaud FI, Whittaker SR, Fischer PM, McClue S, Walton MI, Barrie SE, Garrett MD, Rogers P, Clarke SJ, Kelland LR, Valenti M, Brunton L, Eccles S, Lane DP, Workman $\mathrm{P}$ (2005) In vitro and in vivo pharmacokinetic-pharmacodynamic relationships for the trisubstituted aminopurine cyclin-dependent kinase inhibitors olomoucine, bohemine and CYC202. Clin Cancer Res 11: 4875-4887

Sausville EA (2002) Complexities in the development of cyclin-dependent kinase inhibitor drugs. Trends Mol Med 8(Suppl 4): S32-S37

Sausville EA, Arbuck SG, Messmann R, Headlee D, Bauer KS, Lush RM, Murgo A, Figg WD, Lahusen T, Jaken S, Jing X, Roberge M, Fuse E, Kuwabara T, Senderowicz AM (2001) Phase I trial of 72-h continuous 
infusion UCN-01 in patients with refractory neoplasms. J Clin Oncol 19: $2319-2333$

Schutte B, Nieland L, van Engeland M, Henfling ME, Meijer L, Ramaekers FC (1997) The effect of the cyclin-dependent kinase inhibitor olomoucine on cell cycle kinetics. Exp Cell Res 236: 4-15

Senderowicz AM, Headlee D, Stinson SF, Lush RM, Kalil N, Villalba L, Hill K, Steinberg SM, Figg WD, Tompkins A, Arbuck SG, Sausville EA (1998) Phase I trial of continuous infusion flavopiridol, a novel cyclindependent kinase inhibitor, in patients with refractory neoplasms. J Clin Oncol 16: 2986-2999

Tan AR, Yang X, Berman A, Zhai S, Sparreboom A, Parr AL, Chow C, Brahim JS, Steinberg SM, Figg WD, Swain SM (2004) Phase I trial of the cyclindependent kinase inhibitor flavopiridol in combination with docetaxel in patients with metastatic breast cancer. Clin Cancer Res 10: 5038-5047

Tang L, Li MH, Cao P, Wang F, Chang WR, Bach S, Reinhardt J, Ferandin Y, Galons H, Wan Y, Gray N, Meijer L, Jiang T, Liang DC (2005) Crystal structure of pyridoxal kinase in complex with roscovitine and derivatives. J Biol Chem 280: 31220 - 31229

Tetsu O, McCormick F (2003) Proliferation of cancer cells despite CDK2 inhibition. Cancer Cell 3: 233-245

Thomas JP, Tutsch KD, Cleary JF, Bailey HH, Arzoomanian R, Alberti D, Simon K, Feierabend C, Binger K, Marnoch R, Dresen A, Wilding G (2002) Phase I clinical and pharmacokinetic trial of the cyclindependent kinase inhibitor flavopiridol. Cancer Chemother Pharmacol 50: $465-472$

Vita M, Abdel-Rehim M, Nilsson C, Hassan Z, Skansen P, Wan H, Meurling L, Hassan M (2005) Stability, pKa and plasma protein binding of roscovitine. I Chromatogr B Analyt Technol Biomed Life Sci 821: 75-80

Whittaker SR, Walton MI, Garrett MD, Workman P (2004) The cyclindependent kinase inhibitor CYC202 (R-roscovitine) inhibits RB phosphorylation, causes loss of cyclin D1 and activates the mitogen activated protein kinase pathway. Cancer Res 64: 262-272 